\title{
The Effect of Visual and Haptic Feedback on Manual and Teleoperated Needle Insertion
}

\author{
Oleg Gerovichev ${ }^{1,3}$, Panadda Marayong ${ }^{2}$, and Allison M. Okamura ${ }^{2,3}$ \\ ${ }^{1}$ Department of Biomedical Engineering \\ ${ }^{2}$ Department of Mechanical Engineering \\ ${ }^{3}$ Engineering Research Center for Computer-Integrated Surgical Systems and Technology \\ Johns Hopkins University, Baltimore, MD \\ \{oleg, panadda, aokamura\} ajhu.edu
}

\begin{abstract}
In this paper, we present a study that evaluates the effect of visual and haptic feedbacks and their relevance to human performance in a needle insertion task. A virtual needle insertion simulator with a four-layer tissue model (skin, fat, muscle, and bone) and haptic feedback is used for perceptual experiments. The task was to detect the puncture of a particular layer using haptic and visual cues provided by the simulation. The results show that the addition of force feedback reduces error in detection of transitions between tissue layers by at least 55\%. The addition of real-time visual feedback (image overlay) improved user performance by at least $87 \%$ in scenarios without force feedback. Presentation of both force and visual feedback improved performance by at least $43 \%$ in comparison to scenarios without feedback. These results suggest that real-time image overlay provides greater improvement in performance than force feedback.
\end{abstract}

\section{Introduction}

Current medical practice relies heavily on the manual dexterity of medical personnel. Cardiac bypass surgery, microsurgery, and percutaneous needle therapy are several examples of procedures where human abilities are exercised to the maximum. In addition, the target area is often occluded, particularly in percutaneous therapy. Robotic and imaging technologies have become important aids to assist medical personnel in these tasks. Fichtinger, et al. identify a need for increased precision in aiming and delivery of needles in nerve block and facet joint injection procedures, and propose robot-assisted mechanisms with CT guidance to alleviate the problem [4]. Chinzei, et al. suggest a surgical assist robot to be used with intraoperative MRI, which offers exceptional soft tissue discrimination, for instrument insertion in procedures like prostate tumor biopsy [2]. Masamune, et al. present a needle insertion system for use with MRI-guided neurosurgery [8]. There are also skill-enhancing systems, not targeted specifically at needle insertion tasks, such as the da Vinci ${ }^{\mathrm{TM}}$ Surgical System (Intuitive Surgical Inc., Mountain View, CA) and the ZEUS ${ }^{\circledR}$ Surgical Robotic System (Computer Motion, Inc., Goleta, CA). These robot-assisted surgical systems offer a novel approach to dexterous manipulation of surgical instruments for minimally invasive surgery. However, the systems above do not address the use of force feedback, which could improve performance of the surgeon. 
While visual information is used whenever possible, clinicians traditionally rely on manual force feedback in visually obscured areas. In this paper, we assess the value of visual and force feedback for improving medical tasks, specifically needle insertion. To our knowledge, no similar studies have been performed in the past, although Kontarinis and Howe [6] performed an experiment in which the user was asked to penetrate a thin membrane with a needle held by a slave manipulator while being given combinations of visual (manipulator exposed/hidden), filtered force, and vibratory feedback at the master manipulator. The result was that a combination of force feedback and vibration reduces reaction time in comparison to visually guided approach in puncture tasks. In addition, there are several needle insertion simulators that used the PHANToM ${ }^{\mathrm{TM}}$ haptic interface (SensAble Technologies, Inc., Woburn, MA) to provide 3-DOF force feedback, but no studies validated an improvement in subject performance $[3,7,9]$. In typical approaches, force models have been created from lumbar needle insertion procedure analysis and programmed into a virtual environment. Another approach used force data acquired during ex vivo needle insertions into a porcine specimen to create the force model [1]. A third approach used materials modeling of mechanical tissue properties and MRI imaging for layer anatomy determination [5].

We propose a needle insertion simulation that would assess the advantages of enhanced feedback to the user to improve performance in dexterous tasks. For the purpose of testing human abilities, we created a multi-layer tissue model that provides an illusion of puncture upon needle insertion. The Impulse Engine 2000 (IE2000 $^{\mathrm{TM}}$ ) (Immersion Corp., San Jose, CA) is a 2-DOF haptic interface that has sufficient capability to perform the task proposed.

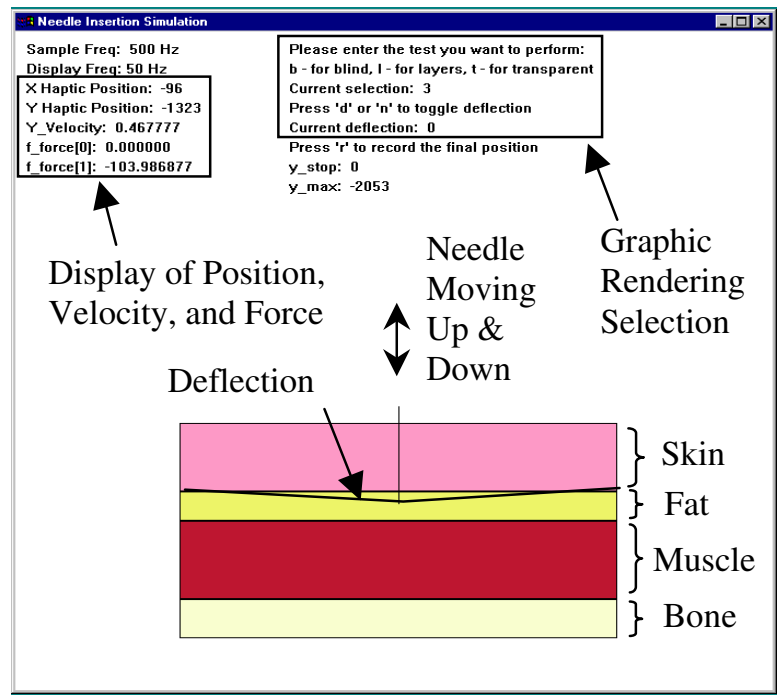

Fig. 1. Needle Insertion Simulation GUI showing layer deflection for visual feedback 


\section{System Design}

\subsection{Haptic Hardware and Software}

The system uses one degree-of-freedom of the IE2000 ${ }^{\mathrm{TM}}$. The simulation was created with Microsoft Visual C++ 6.0 and IE2000 ${ }^{\mathrm{TM}}$ SDK 4.2 on a Pentium III-based system with a Windows NT operating system. The servo loop ran at $500 \mathrm{~Hz}$. The graphics were updated at $50 \mathrm{~Hz}$. The simulation provides the user with different graphical and haptic rendering of multi-layered tissues composed of skin, fat, muscle, and bone layers. We recorded position, velocity and force information during task execution. A screenshot of GUI is shown in Figure 1.

\subsection{Force Model}

Force feedback to the user was based on stiffness force before puncture and damping force during needle translation through the tissue layers. As the needle penetrates deeper into the layers, the user feels the total damping force accumulated from the above tissue layers. Each tissue has different stiffness and damping coefficients, as shown in Table 1. Nominal stiffness coefficients were assigned to reflect differences in tissue densities, and nominal damping coefficients were assigned to reflect the friction between the tissue and the needle shaft. We considered the bone as a solid structure that has a very high stiffness and no viscosity. We implemented the following force model:

$\begin{array}{lll}\text { Skin: } & \text { Upon puncture }=> & F=k_{s} y \\ & \text { After puncture }=> & F=b_{s} y v \\ \text { Fat: } & \text { Upon puncture }=> & F=k_{f} y+b_{s} y_{s} v \\ & \text { After puncture }=> & F=\left(b_{s} y_{s}+b_{f} y\right) v \\ \text { Muscle: } & \text { Upon puncture }=> & F=k_{m} y+\left(b_{s} y_{s}+b_{f} y_{f}\right) v \\ & \text { After puncture }=> & F=\left(b_{s} y_{s}+b_{f} y_{f}+b_{m} y\right) v \\ \text { Bone: } & \text { Upon puncture }=> & F=k_{b} y+\left(b_{s} y_{s}+b_{f} y_{f}+b_{m} y_{m}\right) v\end{array}$

where $k_{s}, k_{f}, k_{m}$, and $k_{b}$ are stiffness coefficients of skin, fat, muscle, and bone, respectively; $b_{s}, b_{f}$, and $b_{m}$ are damping coefficients per unit thickness of skin, fat, and muscle, respectively; $y_{s}, y_{f}$, and $y_{m}$ are thicknesses of each layer; $y$ is the amount of needle penetration with respect to the current layer boundary; and $v$ is needle velocity.

Table 1. Simulation Parameters

\begin{tabular}{|c|c|c|}
\hline Tissue Type & $\begin{array}{c}\text { Stiffness Coefficient (k), } \\
\mathbf{N} / \mathbf{m}\end{array}$ & $\begin{array}{c}\text { Damping Coefficient per unit } \\
\text { length }(\mathbf{b}) \mathbf{~} \mathbf{N} \cdot \mathbf{s} / \mathbf{m}^{\mathbf{2}}\end{array}$ \\
\hline Skin & 331 & 3 \\
\hline Fat & 83 & 1 \\
\hline Muscle & 497 & 3 \\
\hline Bone & 2483 & 0 \\
\hline
\end{tabular}


Table 2. Test Conditions ( $\mathrm{L}=$ Layers, $\mathrm{N}=$ Needle, $\mathrm{D}=$ Deflection, $\mathrm{F}=$ Force $)$

\begin{tabular}{|r|l|c|c|c|c|}
\hline \multicolumn{1}{|c|}{ Test } & \multicolumn{1}{|c|}{ Simulation } & L & N & D & F \\
\hline 1 & Real-time image overlay and needle tracking for tele-insertion & $\mathrm{X}$ & $\mathrm{X}$ & $\mathrm{X}$ & \\
\hline 2 & $\begin{array}{l}\text { Real-time needle tracking for tele-insertion with force feedback } \\
\text { and pre-op image overlay (or manual insertion) }\end{array}$ & $\mathrm{X}$ & $\mathrm{X}$ & & $\mathrm{X}$ \\
\hline 3 & $\begin{array}{l}\text { Real-time image overlay and needle tracking for tele-insertion } \\
\text { with force feedback (or manual insertion) }\end{array}$ & $\mathrm{X}$ & $\mathrm{X}$ & $\mathrm{X}$ & $\mathrm{X}$ \\
\hline 4 & $\begin{array}{l}\text { Insertion based on anatomical knowledge with preoperative } \\
\text { image overlay }\end{array}$ & $\mathrm{X}$ & & \\
\hline 5 & $\begin{array}{l}\text { Tele-insertion based on anatomical knowledge with real-time } \\
\text { image overlay }\end{array}$ & $\mathrm{X}$ & $\mathrm{X}$ & \\
\hline 6 & $\begin{array}{l}\text { Tele-insertion based on anatomical knowledge with pre-op image } \\
\text { overlay and force feedback (or manual insertion) }\end{array}$ & $\mathrm{X}$ & & $\mathrm{X}$ \\
\hline 7 & $\begin{array}{l}\text { Tele-insertion based on anatomical knowledge with real-time } \\
\text { image overlay and force feedback (or manual insertion) }\end{array}$ & $\mathrm{X}$ & $\mathrm{X}$ & $\mathrm{X}$ \\
\hline 8 & $\begin{array}{l}\text { Tele-insertion based on anatomical knowledge with force feed- } \\
\text { back }\end{array}$ & & & $\mathrm{X}$ \\
\hline 9 & Manual insertion in inaccessible area with poor/no visibility & & & $\mathrm{X}$ \\
\hline
\end{tabular}

\section{Experimental Procedure}

Perceptual experiments were conducted with 10 volunteer subjects aged 21-31, 8 of them male and 2 female (Group I). On average, the subjects had medium exposure to haptic interfaces and virtual environments in the past and low exposure to real needle insertion. We also conducted experiments with 4 volunteer male subjects aged 26-40 that had extensive experience with real needle insertion through medical training or research, but no experience with haptic devices (Group II). We presented a training simulation to the subjects in the beginning of the experiment to get them familiar with the mechanics of IE $2000^{\mathrm{TM}}$, introduce different insertion scenarios and allow them to find a comfortable position for joystick operation.

The actual experiments consisted of three trials of nine tests each. In each trial, the tests were presented in random order to minimize learning effects. The goal of each test was to successively detect the transition between the layers either due to haptic or visual cues. For each test, the subject was asked to insert the "virtual" needle into the skin, move from skin into fat, and from fat into muscle. After each transition the subject was to stop the movement of the joystick as soon as possible and allow the experimenter to record appropriate data, then extract the needle from the tissue completely. The data recorded was the penetration depth into the layer after puncture with respect to the layer boundary.

Each of nine presented tests simulated a different needle insertion environment. The conditions for each test are outlined in Table 2. "Deflection" refers to graphical deflection of layer boundary prior to puncture due to the needle. In test 8, subjects could only see the skin boundary, but none of the internal boundaries, whereas in test 9, the subjects were blindfolded and relied completely on force feedback. Tests 4, 5, 6 , and 7 require some anatomical knowledge on the part of the user since they mimic situations where the imaging (a) provides real-time visual feedback, but does not capture the needle in the imaging plane, or (b) is not real time. 


\section{$4 \quad$ Results and Discussion}

To evaluate subject performance, we calculated the error between the penetration depth and the layer boundary for skin, fat, and muscle in each test. We then averaged the error over three trials for each subject. Afterwards, we averaged the resulting errors over all subjects for skin, fat, and muscle in each test. Then, all values were converted from counts into millimeters. Finally, we grouped the results by the following criteria:

1. Group of tests where force feedback was always present and visual cues differed. This studies the effect of imaging on manual insertion or teleoperated tasks (with force feedback).

2. Group of tests where force feedback was always absent and visual cues differed. This studies the effect of imaging on teleoperated tasks.

3. Group of tests where visual feedback was limited and force feedback differed. This studies the effect of force feedback on insertion tasks with or without limited visual feedback.

\subsection{Force Feedback with Varying Visual Feedback}

Figure 2 shows the results of experiments where the subjects received force feedback, yet visual feedback was different in each test (tests 2, 3, and 6-9 in Table 2). As compared to experiment 8 (case "no visual" in Figure 2) when no visual feedback was present, we observed that subjects in Group I (regular subjects) had a $43 \%$ reduction in error $\left(\mathrm{t}_{18}=1.365, \mathrm{p}<0.09\right)$ in tests with fat layer puncture when only the needle was rendered (cases "ND,WN," "WD,WN," and "WD,NN" in Figure 2), but a $67 \%$ reduction in error $\left(\mathrm{t}_{18}=2.586, \mathrm{p}<0.01\right)$ when only the deflection was rendered. Group II (medical subjects) showed at least a $45 \%$ reduction in error $\left(\mathrm{t}_{6}=0.889, \mathrm{p}<0.21\right)$ when only the needle was rendered and $75 \%$ reduction in error $\left(\mathrm{t}_{18}=1.671, \mathrm{p}<0.08\right)$ when only the deflection was added. Skin and muscle trials did not show a significant reduction in error. This shows that in low-level force display scenarios (e.g. fat), users greatly benefit from presence of visual feedback based on real-time tissue boundary tracking, whereas in high-force displays (e.g. skin, muscle), force rendering is sufficient. We observed similar performance and error reduction from the two groups, indicating that performance with force feedback is independent of prior needle insertion experience.

\subsection{No Force Feedback with Varying Visual Feedback}

Figure 3 shows the effect of visual feedback on the performance of the subjects when force feedback is absent. For this set of tests (tests 1, 4, and 5 in Table 2), layer boundaries were rendered, which is equivalent to overlay of pre-operative image data.

When the deflection of the boundary layer and rendering of the needle were absent, the subjects performed badly for all tissue types. Clearly, without any force feedback and needle rendering, the subject has to guess about the location of the needle based on their abstract spatial representation of the needle with respect to the boundaries (anatomical knowledge). As compared to the experiment with needle and layer de- 
flection absent, subjects in Group I showed a 91\% reduction in error $\left(\mathrm{t}_{18}=5.683\right.$, $\mathrm{p}<0.0001)$, and Group II showed a $96 \%$ reduction in error $\left(\mathrm{t}_{6}=3.9, \mathrm{p}<0.004\right)$ with the addition of deflection rendering. With the addition of both deflection and needle rendering, subjects in Group I showed an 87\% reduction in error $\left(\mathrm{t}_{18}=6.061, \mathrm{p}<0.0001\right)$, and Group II showed a 95\% reduction in error $\left(\mathrm{t}_{6}=3.839, \mathrm{p}<0.004\right)$ as compared to the experiment with needle and layer deflection absent. Therefore, boundary layer deflection is a sufficient aid to improve performance, possibly more important than the additional visibility of the needle. This result indicates that the use of real time image overlay will improve insertion depth accuracy.

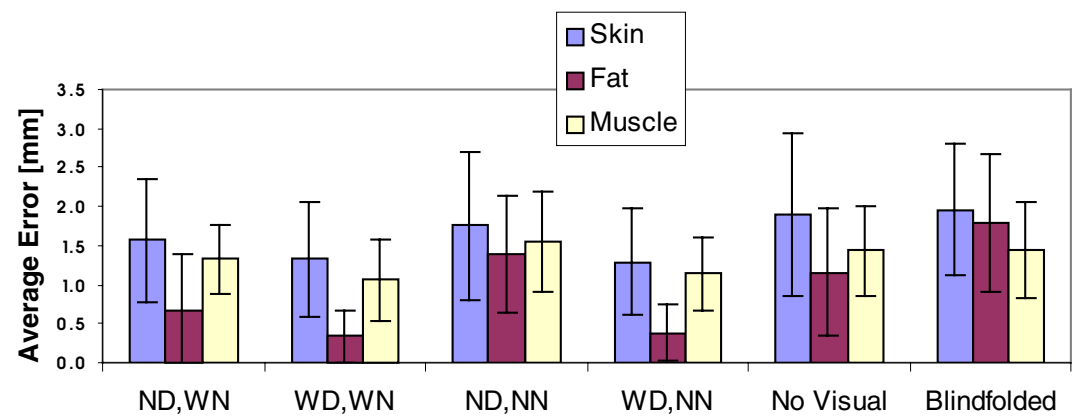

(a) Group I

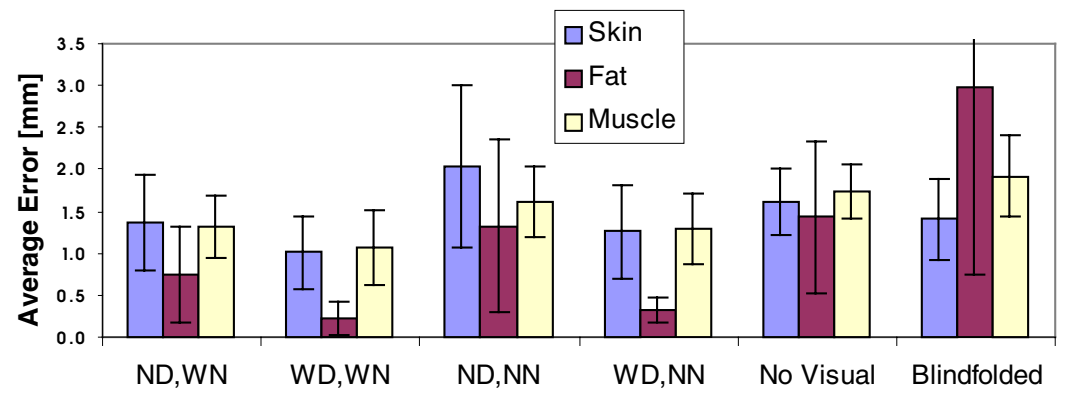

(b) Group II

Fig. 2. Average error in millimeters when force feedback is present with different types of visual feedback where ND $=$ No Deflection, $\mathrm{WD}=$ With Deflection, $\mathrm{NN}=$ No Needle, and WN $=$ With Needle for a) Group I, b) Group II

\subsection{Limited Visual Feedback with Varying Force Feedback}

In Figure 4, visual feedback was limited to tissue boundaries (no deflection and no needle) in each experiment (tests 4, 6, and 8 in Table 2), and no visual feedback at all in one case (test 9 in Table 2). The addition of force feedback reduced the error in Group I by at least $62 \%\left(\mathrm{t}_{18}=3.257, \mathrm{p}<0.004\right)$ as compared to the no force feedback case, and Group II showed at least a 55\% reduction in error $\left(\mathrm{t}_{6}=1.613, \mathrm{p}<0.07\right)$. This shows that addition of force feedback to telesurgical systems could improve surgical performance in situations where vision is occluded. We also observe that the visibility of tissue boundaries has little effect on performance in this scenario because experi- 
ments in which tissue layers alone were visible showed little difference in error from experiments where the tissue layers were invisible.

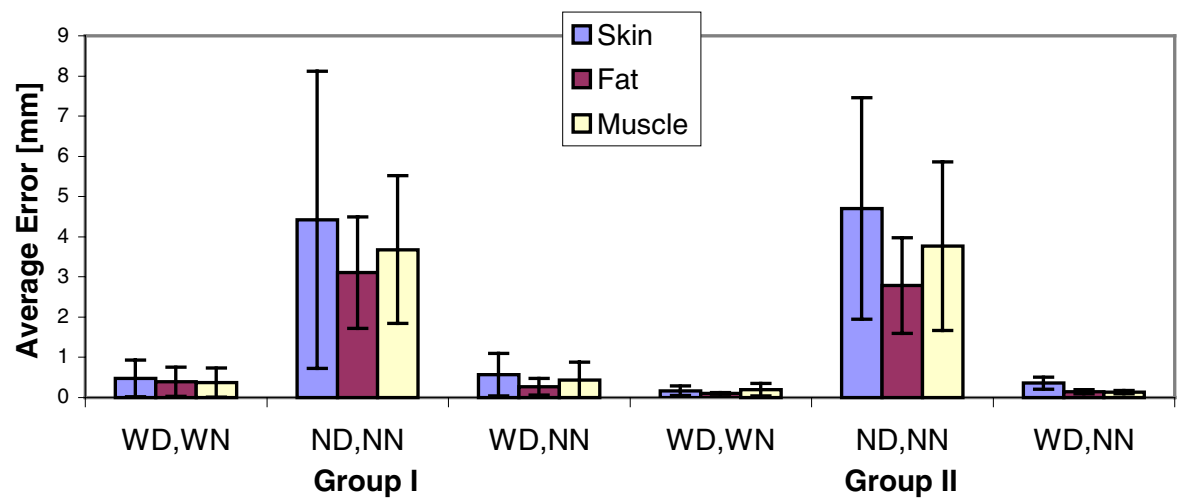

Fig. 3. Average error in millimeters when force feedback is absent with different types of visual feedback where ND $=$ No Deflection, $W D=$ With Deflection, $N N=$ No Needle, and WN $=$ With Needle.

The larger errors in tests with force feedback are due to overshoot after puncture. When the user pushes against a boundary that is suddenly removed, he or she is not able to stop immediately. To prevent this particular effect, a teleoperated robot with no force feedback may be most appropriate. In general, the results demonstrate that visual feedback alone is a more effective aid than haptic feedback alone. While a teleoperated procedure (where no force feedback is provided) could benefit from addition of force feedback, better performance can be obtained from real-time image overlay for manual or teleoperated needle insertions.

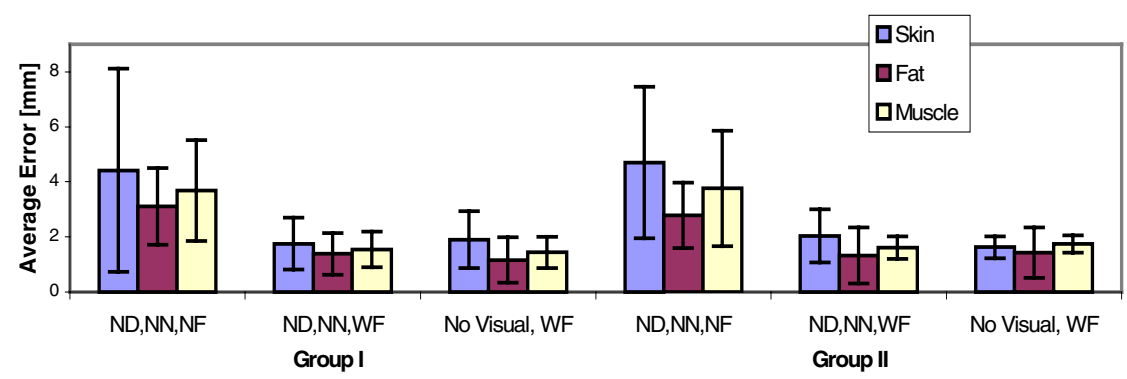

Fig. 4. Average error in millimeters when visual feedback is limited with/without force feedback where $\mathrm{NN}=$ No Needle, $\mathrm{WN}=$ With Needle, NF $=$ No Force, and WF $=$ With Force

\section{Conclusion}

We created a virtual needle insertion simulator with visual and force feedback, and performed a series of perceptual experiments to evaluate performance in a needle insertion task. Users experienced different combinations of visual and haptic feedback, mimicking manual or teleoperated scenarios with and without image overlay 
(real time or pre-operative). The results show that the addition of force feedback to systems that obstruct field of view reduces error in the detection of transitions between tissue layers by at least 55\%. The addition of real-time visual feedback (image overlay) improved user performance by at least $87 \%$ in scenarios without force feedback. Presentation of both force and visual feedback improved performance by at least $43 \%$ in comparison to scenarios without any feedback. Even though haptic feedback provides some improvement, we conclude that graphical display of the deflection of tissue boundaries has a dominating influence on performance. This prompts the use of real-time imaging in medical procedures to provide the user with image overlay to assist in task visualization.

We plan to perform a set of experiments to obtain force profiles for real needle insertions and fit appropriate force models for more realistic simulations. It is also desirable to have experiments for different areas of the body to serve a variety of training needs. In addition, a haptic device that allows the same hand movement as in real needle insertion would provide more realistic simulation, as well as allow training of medical personnel without the use of cadavers or artificial materials.

\section{References}

1. Brett, P. N., Harrison, A. J., Thomas, T. A., "Schemes for the Identification of Tissue Types and Boundaries at the Tool Point for Surgical Needles," IEEE Transactions on Information Technology in Biomedicine, Vol. 4, No. 1, pp. 30-36, 2000.

2. Chinzei, K., Hata, N., Jolesz, A., Kikinis R., "Surgical Assist Robot for the Active Navigation in the Intraoperative MRI: Hardware Design Issues," Proceedings of the IEEE/RSJ International Conference on Intelligent Robots, Vol. 1, pp. 727-732, 2000.

3. Dang, T., Annaswamy, T.M., Srinivasan, M. A., "Development and Evaluation of an Epidural Injection Simulator with Force Feedback for Medical Training," Studies in Health Technology and Informatics, Vol. 29, pp. 564-579, 1996.

4. Fichtinger, G., Masamune, K., Patriciu, A., Tanacs, A., Anderson, J.H., DeWees, T.L., Taylor, R., Stoianovici, D., "Robotically Assisted Percutaneous Local Therapy and Biopsy," The 10th International Conference on Advanced Robotics, pp. 133-151, 2001.

5. Hiemenz Holton, Leslie L., "Force Models for Needle Insertion Created from Measured Needle Puncture Data," Medicine Meets Virtual Reality, pp. 180-186, 2001.

6. Kontarinis, D.A. and Howe, R.D., "Tactile display of vibratory information in teleoperation and virtual environments," Presence, Vol. 4, pp. 387-402, 1995.

7. Kwon, D. S., Kyung, K. U., Kwon, S.M., Ra, J. B., Park, H.W., Kang, H. S., Zeng, J., Cleary, K.R., "Realistic Force Reflection in a Spine Biopsy Simulator," Proceedings of the IEEE International Conference on Robotics and Automation, pp. 1358-1363, 2001.

8. Masamune, K., Kobayashi, E., Masutani, Y., Suzuki, M., Dohi, T., Iseki, H., Takakuro, K., "Development of an MRI-compatible needle insertion manipulator for stereotactic neurosurgery," Journal of Image Guided Surgery, Vol. 1, No. 4, pp. 242-248, 1995.

9. Ra, J. B., et al. A Visually Guided Spine Biopsy Simulator with Force Feedback, SPIE International Conference on Medical Imaging, pp. 36-45, 2001. 